\title{
Multipole Expansion for Relativistic Coulomb Excitation
}

\author{
H. Esbensen \\ Physics Division, Argonne National Laboratory, Argonne, Illinois 60439 \\ C. A. Bertulani \\ Physics Department, Brookhaven National Laboratory, Upton, NY 11973-5000 - USA
}

(October 26, 2018)

\begin{abstract}
We derive a general expression for the multipole expansion of the electromagnetic interaction in relativistic heavy-ion collisions, which can be employed in higher-order dynamical calculations of Coulomb excitation. The interaction has diagonal as well as off-diagonal multipole components, associated with the intrinsic and relative coordinates of projectile and target. A simple truncation in the off-diagonal components gives excellent results in first-order perturbation theory for distant collisions and for beam energies up to $200 \mathrm{MeV} /$ nucleon.
\end{abstract}

PACS number(s): 25.70.-z, 25.70.De

Typeset using REVTEX 


\section{INTRODUCTION}

The Coulomb excitation of nuclei at relativistic energies is commonly calculated in firstorder perturbation theory, using the formalism developed in Ref. [1]. The derivation is restricted to distant collision, where the intrinsic radial coordinates are smaller than the minimum distance between the colliding nuclei. In calculations of the Coulomb dissociation of proton halo nuclei, it is of interest also to consider close collisions, since the density of the valence proton can extend to very large distances. Moreover, higher-order processes may

also play a role, as suggested by the non-relativistic calculations of the ${ }^{8} \mathrm{~B}$ breakup reported in Ref. [2].

In order to calculate the Coulomb dissociation of halo nuclei at energies where relativistic and higher-order effects cannot be ignored, one would need realistic multipole form factors for the electro-magnetic interaction. Such form factors have recently been studied [3] but they were only determined in the long-wave-length limit for distant collisions. We therefore find it timely to study the multipole decomposition of the interaction at relativistic energies, both for close and distant collisions. The form factors we derive can be applied in higher-order dynamical calculations of two-body breakup reactions, such as the continuum discretized coupled-channels calculations [4], or in numerical methods that evolve the two-body wave function in coordinate space [5-7].

We base our study on the so-called Liénard-Wiechert potential and include the effect of the convection current. The magnetization current, on the other hand, is ignored for simplicity. We assume that the relative velocity of the interacting nuclei is a constant, so that the retardation effect associated with a time-dependent velocity can be ignored.

The multipole expansion of the interaction contains both diagonal and off-diagonal multipole components. The off-diagonal components appear because of the Lorentz contraction. Our approach is similar to the method developed by Baltz et al. [8] in their study of the $e^{+} e^{-}$pair production in relativistic heavy-ion collisions. We extend their study and derive the multipole fields that are relevant to Coulomb excitation. 
The multipole expansion of the Liénard-Wiechert potential is presented in Sect. II. There we also discuss how to calculate the interaction associated with the convection current. We propose in Sect. III a simple truncation in the the sum over off-diagonal multipole components. We test this approximation in Sect. IV in first-order perturbation theory for distant collisions by comparing to the exact results of Ref. [1]. Sect. V contains our conclusions.

\section{ELECTRO-MAGNETIC INTERACTION WITH FAST CHARGED PARTICLE}

The electro-magnetic interaction of a fast charged particle (with atomic number $Z_{1}$ ) and a weakly bound proton in a target nucleus has the form [1]

$$
V_{\mathrm{em}}\left(\mathbf{r}, \mathbf{r}^{\prime}\right)=Z_{1} e^{2}\left(\phi-\frac{\mathbf{v}}{2 m c^{2}}[\hat{\mathbf{p}} \phi+\phi \hat{\mathbf{p}}]\right)
$$

The charged particle is assumed to move with constant velocity $\mathbf{v}$ in the z-direction on the straight-line trajectory $\mathbf{r}^{\prime}=\mathbf{b}+\mathbf{v} t$, with impact parameter $\mathbf{b}$ with respect to the target nucleus. The coordinates of the proton in the target nucleus are denoted by $\mathbf{r}$, and $\hat{\mathbf{p}}$ is the conjugate momentum operator. The potential $\phi$ is the Liénard-Wiechert potential

$$
\phi=\frac{\gamma}{\sqrt{\left|\mathbf{r}_{\perp}-\mathbf{r}_{\perp}^{\prime}\right|^{2}+\gamma^{2}\left(z-z^{\prime}\right)^{2}}},
$$

where $\gamma=1 / \sqrt{1-\beta^{2}}$, and $\beta=v / c$. The second part of the interaction (1), which contains the momentum operator $\hat{\mathbf{p}}$, is due to the convection current. We ignore the magnetization current but it could be included by replacing the momentum operator by $\hat{\mathbf{p}}+\hbar \nabla \times \boldsymbol{\mu}_{p}$, where $\boldsymbol{\mu}_{p}$ is the magnetic moment of the proton.

In the two-body breakup of a target nucleus, into a proton and a core nucleus, one would actually have to consider the effect of two interactions with the projectile, namely, the 'direct' interaction with the proton and the 'recoil' interaction with the core. We will not discuss this complication here, since the multipole expansion of the two interactions can easily be generated from the formulation given below. 


\section{A. Multipole expansion}

The multipole expansion of the Liénard-Wiechert potential $\phi$ can be obtained from the Fourier representation [8]

$$
\phi=\frac{4 \pi}{(2 \pi)^{3}} \int d \mathbf{q} \frac{e^{i \mathbf{q} \cdot\left(\mathbf{r}-\mathbf{r}^{\prime}\right)}}{q_{\perp}^{2}+q_{z}^{2} / \gamma^{2}}=\frac{4 \pi}{(2 \pi)^{3}} \int d \mathbf{q} \frac{e^{i \mathbf{q} \cdot\left(\mathbf{r}-\mathbf{r}^{\prime}\right)}}{q^{2}} \frac{1}{1-\beta^{2} \cos ^{2}\left(\theta_{q}\right)} .
$$

Except for the last factor, this is just the ordinary Coulomb potential. To proceed, we introduce the multipole expansion of the last term in Eq. (3)

$$
\frac{1}{1-\beta^{2} \cos ^{2}(\theta)}=\sum_{\lambda \text { even }} g_{\lambda}(\beta) P_{\lambda}\left(\cos \left(\theta_{q}\right)\right)
$$

where

$$
g_{\lambda}(\beta)=(2 \lambda+1) \beta^{-1} Q_{\lambda}\left(\beta^{-1}\right),
$$

and

$$
Q_{\lambda}(z)=\frac{1}{2} \int_{-1}^{1} d t \frac{P_{\lambda}(t)}{z-t}
$$

are Legendre polynomials of the second kind; cf. Eq. 8.825 of Ref. [9] or Eq. (8.8.3) of Ref. [10]. Explicit expressions and recursion relations for $Q_{\lambda}(z)$ are given in Ref. [10].

We can now insert the plane wave expansion

$$
e^{i \mathbf{q} \cdot \mathbf{r}}=4 \pi \sum_{l m} i^{l} j_{l}(q r) Y_{l m}(\hat{q}) Y_{l m}^{*}(\hat{r})
$$

for the two plane waves in Eq. (3) and obtain

$$
\phi=4 \pi \sum_{l m l^{\prime} m^{\prime}} i^{l-l^{\prime}} R_{l l^{\prime}}\left(r, r^{\prime}\right) A_{l m, l^{\prime} m^{\prime}}(\beta) Y_{l m}^{*}(\hat{r}) Y_{l^{\prime} m^{\prime}}\left(\hat{r}^{\prime}\right),
$$

where

$$
R_{l, l^{\prime}}\left(r, r^{\prime}\right)=\frac{2}{\pi} \int_{0}^{\infty} d q j_{l}(q r) j_{l^{\prime}}\left(q r^{\prime}\right)=\frac{1}{\sqrt{r r^{\prime}}} \int_{0}^{\infty} \frac{d q}{q} J_{l+1 / 2}(q r) J_{l^{\prime}+1 / 2}\left(q r^{\prime}\right),
$$

and

$$
A_{l m, l^{\prime} m^{\prime}}(\beta)=\left\langle Y_{l^{\prime} m^{\prime}}\left|\frac{1}{1-\beta^{2} \cos ^{2}\left(\theta_{q}\right)}\right| Y_{l m}\right\rangle=\sum_{\lambda \text { even }} g_{\lambda}(\beta)\left\langle Y_{l^{\prime} m^{\prime}}(\hat{q})\left|P_{\lambda}\left(\theta_{q}\right)\right| Y_{l m}(\hat{q})\right\rangle .
$$


The above expressions were derived for a trajectory with constant velocity in the zdirection. The matrix (5b) is therefore diagonal in $m$. Let us also give a more general result which does not refer to any specific coordinate system. This can be done by noticing that the angle $\theta_{q}$ in Eq. (5b) is actually the angle between $\mathbf{q}$ and the velocity $\mathbf{v}$. Thus by inserting $P_{\lambda}\left(\theta_{q}\right)=\sum_{\mu} D_{\mu 0}^{\lambda}(\hat{v})^{*} D_{\mu 0}^{\lambda}(\hat{q})$ we obtain

$$
A_{l m, l^{\prime} m^{\prime}}(\beta, \hat{v})=\sum_{\lambda \text { ever }} g_{\lambda}(\beta) \sum_{\mu}\left(D_{\mu 0}^{\lambda}(\hat{v})\right)^{*}\left\langle l m \lambda \mu \mid l^{\prime} m^{\prime}\right\rangle\left\langle l^{\prime} 0 \lambda 0 \mid l 0\right\rangle,
$$

where the last two Clebsch-Gordan coefficients is the matrix element $\left\langle Y_{l^{\prime} m^{\prime}}\left|D_{\mu 0}^{\lambda}\right| Y_{l m}\right\rangle$.

We note that the expansion (5) contains diagonal (i.e. $l=l^{\prime}$ ) as well as off-diagonal multipole components (i. e. $(l m) \neq\left(l^{\prime} m^{\prime}\right)$. The off-diagonal components are caused by the Lorentz contraction which destroys the spherical symmetry of the potential $\phi$. The radial dependence is determined by the integral (5a), and analytic expressions and recursion relations are derived in App. A. In numerical applications one can include the finite size of the projectile simply by multiplying the integrand in Eq. (5a) by the Fourier transform of the charge distribution. However, we find it instructive to show the analytic expressions for point-particles.

The radial dependence of the diagonal components is determined by (see Eq. (A.5))

$$
R_{l, l}\left(r, r^{\prime}\right)=\frac{1}{2 l+1} \frac{r_{<}^{l}}{r_{>}^{l+1}}
$$

where $r_{<}=\min \left(r, r^{\prime}\right)$ and $r_{>}=\max \left(r, r^{\prime}\right)$. This is identical to the dependence one has in the non-relativistic limit. In fact, the well-known multipole expansion of the Coulomb interaction is recovered from Eq. (5), when we insert the non-relativistic limit of Eq. (5b), viz. $A_{l m, l^{\prime} m^{\prime}}(\beta=0)=\delta_{l l^{\prime}} \delta_{m m^{\prime}}$.

The off-diagonal multipole contributions to $\phi$ are more complicated. The radial form factors have the symmetry property

$$
R_{l, l^{\prime}}\left(r, r^{\prime}\right)=R_{l^{\prime}, l}\left(r^{\prime}, r\right)
$$

According to Eq. (5b), we only need expressions for even values of $\left|l-l^{\prime}\right|$. We show in App. 
A that

$$
R_{l, l+\Lambda}\left(r, r^{\prime}\right)=0, \quad \text { for } r \geq r^{\prime} \text { and } \Lambda=2,4, \ldots
$$

The explicit, non-zero expressions can be obtained from Eq. (A.4). For $\Lambda=2$ we obtain in particular (see Eq. (A.5))

$$
R_{l, l+2}\left(r, r^{\prime}\right)=\frac{1}{2} \frac{r^{l}}{r^{\prime l+1}}\left[1-\left(\frac{r}{r^{\prime}}\right)^{2}\right], \text { for } r<r^{\prime} .
$$

One can exploit the properties (7a) and (7b) and write expressions for $\phi$ that are valid for distant and close collisions, respectively. Thus we obtain for distant collisions $\left(r<r^{\prime}\right)$

$$
\phi_{\text {dist }}=\sum_{l m m^{\prime}} 4 \pi Y_{l m}^{*}(\hat{r}) \sum_{\Lambda=0,2, .} i^{\Lambda} R_{l, l+\Lambda}\left(r, r^{\prime}\right) A_{l m, l+\Lambda m^{\prime}} Y_{l+\Lambda, m^{\prime}}\left(\hat{r}^{\prime}\right) .
$$

For close collisions $\left(r>r^{\prime}\right)$ we can use the expression

$$
\phi_{\text {close }}=\sum_{l m m^{\prime}} 4 \pi Y_{l m}^{*}(\hat{r}) \sum_{\Lambda=0,2, .} i^{\Lambda} R_{l, l-\Lambda}\left(r, r^{\prime}\right) A_{l m, l-\Lambda m^{\prime}} Y_{l-\Lambda, m^{\prime}}\left(\hat{r}^{\prime}\right) .
$$

The sum over $\Lambda$ is finite for close collisions, since $l-\Lambda$ must be non-negative. This is a very nice feature, which makes it feasible to include all terms. For distant collisions, on the other hand, one would have to make a truncation in the sum over $\Lambda$.

\section{B. Convection current interaction}

The contribution to the interaction (1) that originates from the convection current is

$$
\phi^{\prime}=\frac{i \hbar}{2 m c^{2}}((\mathbf{v} \cdot \boldsymbol{\nabla}) \phi+\phi(\mathbf{v} \cdot \boldsymbol{\nabla}))=\frac{i \hbar}{2 m c^{2}}(\mathbf{v} \cdot(\boldsymbol{\nabla} \phi)+2 \phi(\mathbf{v} \cdot \boldsymbol{\nabla})) .
$$

The operator $\mathbf{v} \cdot \boldsymbol{\nabla}$ is effectively of dipole nature, as shown in App. B. In practical numerical calculations it will operate on an expression of the form $r^{-1} f(r) Y_{L M}(\hat{r})$. ¿From Eq. (B.4) we then obtain

$$
\begin{gathered}
\mathbf{v} \cdot \nabla\left(\frac{f(r)}{r} Y_{L M}(\hat{r})\right)=v \sum_{\mu}\left(D_{\mu 0}^{1}(\hat{v})\right)^{*} \sum_{L^{\prime} M^{\prime}} Y_{L^{\prime} M^{\prime}}(\hat{r})\left\langle Y_{L^{\prime} M^{\prime}}\left|D_{\mu 0}^{1}\right| Y_{L M}\right\rangle \\
\times\left(\frac{1}{r} \frac{d f(r)}{d r}-\frac{f(r)}{2 r^{2}}\left[L^{\prime}\left(L^{\prime}+1\right)-L(L+1)\right]\right) .
\end{gathered}
$$


We can also give an explicit expression for the term $c^{-1} \mathbf{v} \cdot(\boldsymbol{\nabla} \phi)$, which appears in second version of Eq. (10). Assuming that $\mathbf{v}$ points in the z-direction, we obtain from Eq. (3)

$$
\beta\left(\frac{d \phi}{d z}\right)=\frac{4 \pi}{(2 \pi)^{3}} \int d \mathbf{q} \frac{e^{i \mathbf{q}\left(\mathbf{r}-\mathbf{r}^{\prime}\right)}}{q^{2}} \frac{i \beta q \cos \left(\theta_{q}\right)}{1-\beta^{2} \cos ^{2}\left(\theta_{q}\right)} .
$$

We can now repeat the derivation that lead to Eq. (5) and obtain

$$
\beta\left(\frac{\partial \phi}{\partial z}\right)=4 \pi i \sum_{l l^{\prime}} i^{l-l^{\prime}} P_{l l^{\prime}}\left(r, r^{\prime}\right) \sum_{m} B_{l l^{\prime} m}(\beta) Y_{l m}^{*}(\hat{r}) Y_{l^{\prime} m}\left(\hat{r}^{\prime}\right),
$$

where

$$
P_{l l^{\prime}}\left(r, r^{\prime}\right)=\frac{2}{\pi} \int_{0}^{\infty} d q q j_{l}(q r) j_{l^{\prime}}\left(q r^{\prime}\right)
$$

and

$$
B_{l l^{\prime} m}(\beta)=\left\langle Y_{l^{\prime} m}\left|\frac{\beta \cos (\theta)}{1-\beta^{2} \cos ^{2}(\theta)}\right| Y_{l m}\right\rangle=\sum_{\lambda \text { odd }} g_{\lambda}(\beta)\left\langle Y_{l^{\prime} m}\left|P_{\lambda}(\cos (\theta))\right| Y_{l m}\right\rangle
$$

The last expression is similar to Eq. (5b) but the sum is now over odd values of $\lambda$. The dependence on the radial coordinates can be derived from Eqs. (A.7-8). We note that matrix

elements of $\phi^{\prime}$ can be calculated in a much simpler way in first-order perturbation theory, as we shall see in Sect. IV.

\section{SIGNIFICANCE OF RELATIVISTIC CORRECTIONS}

In order to illustrate the significance of relativistic corrections at intermediate energies, we show in Fig. 1 the functions $g_{\lambda}(\beta)$ defined in Eq. (4b). They are seen to decrease rapidly as function of $\lambda$ when $(v / c)^{2}<0.5$. This suggests that one would only need a few terms in the sum over $\Lambda$ in Eq. (9a).

In the next section we determine the range of velocities where a truncation to $\Lambda=0,2$ is reasonable for distant collision. Thus we will apply the approximation

$$
\begin{gathered}
\phi_{l m}^{\text {dist }} \approx \frac{4 \pi}{2 l+1} \frac{r^{l}}{r^{\prime l+1}} Y_{l m}^{*}(\hat{r}) \\
\times \sum_{m^{\prime}}\left[A_{l m, l m^{\prime}}(\beta) Y_{l m^{\prime}}\left(\hat{r}^{\prime}\right)-\frac{2 l+1}{2} A_{l m, l+2, m^{\prime}}(\beta) Y_{l+2, m^{\prime}}\left(\hat{r}^{\prime}\right)\left(1-\left(r / r^{\prime}\right)^{2}\right)\right] .
\end{gathered}
$$


We assume a straight line trajectory with constant velocity in the z-direction and calculate the Coulomb excitation in first-order perturbation theory. We can then test the accuracy of the truncation, Eq. (13), by comparing to the exact results from Ref. [1].

\section{TEST IN FIRST-ORDER PERTURBATION THEORY}

In first-order perturbation theory for distant collisions one needs the Fourier integral of the multipole fields,

$$
\phi_{l m}(\mathbf{r}, \omega)=\int_{-\infty}^{\infty} d t e^{i \omega t} \phi_{l m}(\mathbf{r}, t)=\sqrt{\frac{4 \pi}{2 l+1}} r^{l} Y_{l m}^{*}(\hat{r}) S_{l m}(\omega)
$$

where $\hbar \omega$ is the excitation energy and $S_{l m}(\omega)$ are the so-called orbital integrals,

$$
S_{l m}(\omega)=\sqrt{\frac{4 \pi}{2 l+1}} \int_{-\infty}^{\infty} d t e^{i \omega t} \frac{Y_{l m}(\hat{R})}{R^{l+1}} .
$$

For non-relativistic Coulomb excitation and a straight-line trajectory, these integrals are [1]

$$
S_{l m}^{\mathrm{NR}}(\omega)=\frac{2}{v} \frac{i^{l+m}}{\sqrt{(l+m) !(l-m) !}}\left(\frac{\omega}{v}\right)^{l} K_{m}(\omega b / v) .
$$

Below we derive the orbital integrals for the truncated interaction (13).

\section{A. Liénard-Wiechert potential}

The Fourier transform (14a) of the multipole field (13) can be expressed in terms of various combinations of the non-relativistic orbital integrals (15). This is obvious for the first term in (13), and the second term is calculated in App. C. A slight complication is the

very last term in Eq. (13) which contains the factor $\left(r / r^{\prime}\right)^{2}$. It turns out to be a factor of $(\omega r / v)^{2}$ smaller than the dominant term so let us ignore it. Thus we obtain

$$
S_{l m}(\omega)=A_{l l m}(\beta) S_{l m}^{\mathrm{NR}}(\omega)-A_{l, l+2, m}(\beta) \frac{2 l+1}{2} \sqrt{\frac{4 \pi}{2 l+1}} \int_{-\infty}^{\infty} d t e^{i \omega t} \frac{Y_{l+2, m}(\hat{R})}{R^{l+1}} .
$$

The latter integral is given in Eq. (C.5). 


\section{B. Convection current interaction}

We also need to calculate the contribution from the convection current, Eq. (10). Here we can again make the substitution (B.1). Assuming that the single-particle potential commutes with $\mathbf{r}$ we can go a step further and replace the momentum operator by $p_{z}=\frac{i m}{\hbar}[H, z]$, where $H$ is the single-particle Hamiltonian. Thus we obtain

$$
\phi^{\prime}=-\frac{i \beta}{2 \hbar c}([H, z] \phi+\phi[H, z])=-\frac{i \beta}{2 \hbar c}([H, z \phi]+\phi H z-z H \phi) .
$$

We note that in first-order perturbation theory one can effectively replace the commutator $[H, z \phi]$ by $\hbar \omega z \phi$. The other term, $\phi H z-z H \phi$, has to be considered more carefully.

Let us write the multipole expansion of $\phi^{\prime}$ as in Eq. (14a),

$$
\phi^{\prime}=\sqrt{\frac{4 \pi}{2 l+1}} r^{l} Y_{l m}(\hat{r}) S_{l m}^{\prime} .
$$

Since $z$ in Eq. (17) is a dipole operator, we see that $\phi_{l m}^{\prime}$ can receive contributions from both $\phi_{l-1, m}$ and $\phi_{l+1, m}$. The contribution from the latter is smaller (by a factor of $(\omega r / v)^{2}$ ) so let us ignore it. Let us first consider the contribution from the commutator $[H, z \phi]$. This leads to the expression

$$
S_{l m}^{\prime}([H, z \phi]) \approx-\frac{i \beta^{2}}{2} \frac{\omega}{v} S_{l-1, m} \frac{\sqrt{l^{2}-m^{2}}}{2 l-1}
$$

For the dipole field we see that the second term in Eq. (17), namely $\phi_{00} H z-z H \phi_{00}$, is identical to the first term because $\phi_{00}$ commutes with the Hamiltonian $H$. Thus we obtain

$$
S_{10}^{\prime} \approx-\beta^{2} \frac{i \omega}{c} S_{00}, \quad S_{11}^{\prime}=0
$$

For the quadrupole field we can neglect the second term of Eq. (17) It is identical to zero when we insert $\phi_{10}$, whereas inserting $\phi_{11}$ leads to a magnetic type transition $\left(\propto\left(l_{x}+i l_{y}\right)\right)$. Thus we will use the approximation

$$
S_{2 m}^{\prime} \approx-\frac{i \beta^{2}}{2} \frac{\omega}{v} S_{1, m} \frac{\sqrt{4-m^{2}}}{3}
$$




\section{Comparison to exact results}

In order to illustrate the orbital integrals we obtain from the approximation (13), it is useful to plot the reduced values

$$
\widetilde{S}_{l m}=\frac{S_{l m}+S_{l m}^{\prime}}{N_{l m}}, \quad \text { where } \quad N_{l m}=\frac{2}{v} \frac{i^{l+m} b^{-l}}{\sqrt{(l+m) !(l-m) !}} .
$$

The results we obtain for dipole and quadrupole excitations are shown by the solid symbols

in Figs. 2 and 3, respectively, as functions of the adiabaticity parameter $\xi=\omega b / v$. Here we have chosen the beam velocity $v / c=0.6$

The full relativistic expression for electric transitions [1], which also includes a contribution from the convection current, is

$$
\widetilde{S}_{l m}^{\mathrm{Rel}}(\omega)=S_{l m}^{\mathrm{Rel}} / N_{l m}=\frac{1}{\gamma} G_{l m}(\beta) \xi^{l} K_{m}\left(\frac{\xi}{\gamma}\right)
$$

where $G_{l m}$ can be extracted from [1],

$$
G_{10}=G_{20}=G_{2 \pm 2}=\frac{1}{\gamma}, G_{1 \pm 1}=1, G_{2 \pm 1}=1-\frac{\beta^{2}}{2}
$$

This result is shown by the solid curves in Figs. 2 and 3. The dashed curves represent the

non-relativistic result $\widetilde{S}_{l m}^{N R}=\xi^{l} K_{m}(\xi)$, c. f. Eq. (15). It is seen that relativistic effects are significant and that the truncated calculation is in very good agreement with the full result. If we choose a larger velocity, say $v / c=0.8$, some discrepancy starts to occur, so it will be necessary to include higher values of $\Lambda$ in the sum (9a).

\section{CONCLUSION}

The multipole expansion of the electro-magnetic interaction in relativistic heavy-ion collisions that we have presented is exact for a straight-line trajectory with constant velocity. It has not previously, as far as we know, been used in calculations of the Coulomb excitation. The formulation is, in particular, applicable to reactions where close collisions play 
a role, and it is also well suited for calculations of higher-order processes in the Coulomb dissociation of halo nuclei at intermediate energies.

The multipole expansion contains diagonal as well as off-diagonal components, associated with the intrinsic coordinates and the coordinates for the relative motion of the colliding nuclei. The off-diagonal multipole components are caused by relativistic effects, essentially by the Lorentz contraction of the Coulomb field; only the diagonal components survive in the non-relativistic limit.

The number of off-diagonal multipole components is finite for close collisions but it is, in principle, infinite for distant collisions. However, it is sufficient to include just a few offdiagonal terms at intermediate energies. Thus we find that the diagonal and first off-diagonal

terms (i. e., $\Lambda=0,2$ in Eq. (9a)) give excellent results in first-order perturbation theory for distant collisions and for velocities up to $v / c \approx 0.6$, i. e. up to about $200 \mathrm{MeV} /$ nucleon.

Acknowledgments This work was supported by the US Department of Energy, Nuclear Physics Division, under Contracts W-31-109-ENG-38 and DE-AC02-98CH10886, and by a fellowship (C.A.B.) from the J.S. Guggenheim Memorial Foundation.

\section{APPENDICES}

\section{A. Bessel function integrals}

Here we study the integral in Eq. (5a)

$$
I_{\mu, \mu+2 m}(a, b)=\int_{0}^{\infty} \frac{d q}{q} J_{\mu}(a q) J_{\mu+2 m}(b q)
$$

where $\mu=l+1 / 2$ and $2 m$ is an even number, according to parity selection of Eq. (5b). This integral is given in many textbooks but we shall mainly refer to Abramowitz [10]. We note that it has the symmetry

$$
I_{\mu+2 m, \mu}(a, b)=I_{\mu, \mu+2 m}(b, a),
$$

so it is sufficient to determine (A.1) for all values of $a$ and $b$. 
Let us first assume that $a<b$. From Eq. 11.4.34 of Ref. [10] we obtain (with $\nu=\mu+2 m$ and $\lambda=1)$

$$
I_{\mu, \mu+2 m}(a, b)=\frac{1}{2}\left(\frac{a}{b}\right)^{\mu} \frac{\Gamma(\mu+m)}{\Gamma(\mu+1) \Gamma(m+1)}{ }_{2} F_{1}\left(\mu+m,-m ; \mu+1 ;(a / b)^{2}\right) .
$$

The hypergeometric function in Eq. (A.3) is a polynomial of degree $m$, which can be obtained directly for the series Eq. 15.1.1 of Ref. [10]. Inserting into that expression the Pochhammer's symbols (see Eq. (6.1.22) of Ref. [10]) we obtain

$$
I_{\mu, \mu+2 m}(a, b)=\frac{1}{2}\left(\frac{a}{b}\right)^{\mu} \sum_{n=0}^{m} \frac{(-1)^{n} \Gamma(\mu+m+n)}{\Gamma(m+1-n) \Gamma(\mu+1+n)} \frac{(a / b)^{2 n}}{n !} .
$$

For $m=0,1$ and 2 we obtain in particular

$$
\begin{aligned}
& I_{\mu, \mu}(a, b)=\frac{1}{2 \mu}(a / b)^{\mu}, \quad I_{\mu, \mu+2}(a, b)=\frac{1}{2}(a / b)^{\mu}\left[1-(a / b)^{2}\right] \\
& I_{\mu, \mu+4}(a, b)=\frac{1}{4}(a / b)^{\mu}\left[1-(a / b)^{2}\right]\left[\mu+1-(\mu+3)(a / b)^{2}\right] .
\end{aligned}
$$

Using the symmetry property (A.2) we see that $I_{\mu, \mu}$ is consistent with Eq. (6).

When $a>b$ and $m$ is non-zero we find that

$$
I_{\mu, \mu+2 m}(a, b)=0, \quad \text { for } a>b, \quad m=1,2, \ldots
$$

This result follows from Eq. 11.4.33 of Ref. [10] because that expression will contain the factor $1 / \Gamma(-m+1)$ which is zero for $m=1,2,3, \ldots$

The functions $P_{l, l^{\prime}}\left(r, r^{\prime}\right)$ defined in Eq. (12a) can be obtained from the relation

$$
P_{l, l^{\prime}+1}\left(r, r^{\prime}\right)+P_{l, l^{\prime}-1}\left(r, r^{\prime}\right)=\frac{2 l^{\prime}+1}{r^{\prime}} R_{l, l^{\prime}}\left(r, r^{\prime}\right) .
$$

This expression follows directly from the definitions in Eqs. (5a) and (12a) and the recursion relation for spherical Bessel functions, $z j_{l-1}(z)+z j_{l+1}(z)=(2 l+1) j_{l}(z)$. For $l^{\prime}=l+1$ we find explicitly (c.f. Eq. 6.575 of Ref. [9])

$$
P_{l, l+1}\left(r, r^{\prime}\right)=P_{l+1, l}\left(r^{\prime}, r\right)=\frac{1}{r^{\prime 2}}\left(\frac{r}{r^{\prime}}\right)^{l}, \quad \text { for } r \leq r^{\prime},
$$

while it is zero when $r>r^{\prime}$. The radial dependence can now in general be derived from the recursion relation (A.7). 


\section{B. Current operator}

Here we derive an expression for the operator $\mathbf{v} \cdot \boldsymbol{\nabla}$, which appears in the contribution from the convection current. It can be expressed in terms of a commutator with the Laplace operator $\Delta$ as follows

$$
\mathbf{v} \cdot \boldsymbol{\nabla}=\frac{1}{2}[\Delta, \mathbf{v} \cdot \mathbf{r}]
$$

Here we can insert

$$
\mathbf{v} \cdot \mathbf{r}=v r \cos \left(\theta_{v r}\right)=v r \sum_{\mu}\left(D_{\mu 0}^{1}(\hat{v})\right)^{*} D_{\mu 0}^{1}(\hat{r}),
$$

and express the Laplace operator in spherical coordinates

$$
\Delta=\frac{d^{2}}{d r^{2}}+\frac{2}{r} \frac{d}{d r}-\frac{\hat{L}^{2}}{r^{2}}
$$

where $\hat{L}$ is the angular momentum operator. Thus we obtain

$$
\mathbf{v} \cdot \boldsymbol{\nabla}=v \sum_{\mu}\left(D_{\mu 0}^{\lambda}(\hat{v})\right)^{*}\left(D_{\mu 0}^{\lambda}(\hat{r})\left(\frac{d}{d r}+\frac{1}{r}\right)-\frac{1}{2 r}\left[\hat{L}^{2}, D_{\mu 0}^{\lambda}(\hat{r})\right]\right) .
$$

\section{Orbital integral}

Here we derive an expression for the integral

$$
\mathcal{S}_{l 2 m}(\omega)=\sqrt{\frac{4 \pi}{2 l+1}} \int_{-\infty}^{\infty} d t e^{i \omega t} \frac{Y_{l+2, m}(\hat{R})}{R^{l+1}},
$$

which appears in Eq (16). This integral can be calculated in a simple way by observing that

$$
Y_{l+2, m}=\sqrt{\frac{2 l+5}{2 l+3}} \sum_{\mu=-1}^{1} C_{\mu}^{1} D_{\mu 0}^{1}(\hat{R}) Y_{l+1, m-\mu}(\hat{R}),
$$

where (use Eq. (14) of App. D in Ref. [11])

$$
\begin{gathered}
C_{0}^{1}=\frac{\sqrt{(l+2+m)(l+2-m)}}{l+2}, \\
C_{-1}^{1}=\frac{1}{\sqrt{2}} \frac{\sqrt{(l+1-m)(l+2-m)}}{l+2}, \\
C_{1}^{1}=\frac{1}{\sqrt{2}} \frac{\sqrt{(l+1+m)(l+2+m)}}{l+2} .
\end{gathered}
$$


The trajectory we consider falls in the $\mathrm{x}-\mathrm{z}$ plane, with $\cos (\theta)=v t / R$ and $\sin (\theta)=b / R$. The integral (C.1) can therefore be expressed in terms of the non-relativistic orbital integrals (15) as follows

$$
\begin{aligned}
& \mathcal{S}_{l 2 m}(\omega)=\sqrt{\frac{2 l+5}{2 l+1}}\left[C_{0}^{1} v\left(-i \frac{d}{d \omega}\right) S_{l+1, m}^{N R}(\omega)\right. \\
& \left.+\frac{b}{\sqrt{2}}\left(C_{-1}^{1} S_{l+1, m+1}^{N R}(\omega)-C_{1}^{1} S_{l+1, m-1}^{N R}(\omega)\right)\right] .
\end{aligned}
$$

Working out the details this can be written explicitly as

$$
\begin{gathered}
\mathcal{S}_{l 2 m}(\omega)=\frac{1}{v} \sqrt{\frac{2 l+5}{2 l+1}} \frac{1}{l+2} \frac{i^{l+2+m}}{\sqrt{(l+2+m) !(l+2-m) !}}\left(\frac{\omega}{v}\right)^{l} \\
{\left[(2 l+3) \xi\left((l+2-m) K_{m+1}(\xi)+(l+2+m) K_{m-1}(\xi)\right)-2(l+1)(l+2+m)(l+2-m) K_{m}(\xi)\right]}
\end{gathered}
$$

where $\xi=\omega b / v$. Or even more compact:

$$
\begin{gathered}
\mathcal{S}_{l 2 m}(\omega)=-\frac{2}{v} \sqrt{\frac{2 l+5}{2 l+1}} \frac{i^{l+2+m}}{\sqrt{(l+2+m) !(l+2-m) !}}\left(\frac{\omega}{v}\right)^{l} \\
{\left[\left((l+1)(l+2)+m^{2}\right) K_{m}(\xi)-\frac{2 l+3}{2} \xi\left(K_{m-1}(\xi)+K_{m+1}(\xi)\right)\right] .}
\end{gathered}
$$




\section{REFERENCES}

[1] A. Winther and K. Alder, Nucl. Phys. A319, 518 (1979).

[2] H. Esbensen and G. F. Bertsch, Nucl. Phys. A600, 37 (1996).

[3] C. A. Bertulani and V. Yu. Ponomarev, Phys. Rep. 321, 139 (1999).

[4] F. M. Nunes and I. J. Thompson, Phys. Rev. C 59, 2652 (1999).

[5] H. Esbensen, G. F. Bertsch, and C. A. Bertulani, Nucl. Phys. A581, 107 (1995).

[6] T. Kido, K. Yabana, and Y. Suzuki, Phys. Rev. C 53, 2296 (1996).

[7] V. S. Melezhik and D. Baye, Phys. Rev. C 593232 (1999).

[8] A. J. Baltz, M. J. Rhodes-Brown, and J. Weneser, Phys. Rev. A 44, 5569 (1991).

[9] I. S. Gradshteyn and I. M. Ryzhik, Tables of Integrals, Series and Products, (Academic Press, New York, 1965).

[10] M. Abramowitz and I. A. Stegun, Handbook of Mathematical Functions (4th printing, December 1965).

[11] K. Alder and A. Winther, Electromagnetic Excitation (North-Holland, 1975). 


\section{FIGURES}

FIG. 1. The functions $g_{\lambda}(v / c)$, defined in Eq. (4b), are shown as functions of $(v / c)^{2}$ for $\lambda=0-6$.

FIG. 2. The reduced values of orbital integrals, defined in Eq. (19), are shown for dipole excitations as functions of the adiabaticity parameter $\xi=\omega b / v$. The beam velocity is $v / c=0.6$. The solid curves are the exact results, Eq. (20), and the dashed curves are the non-relativistic results. The solid symbols are the results of our approximation discussed in Sect. IV.

FIG. 3. Similar to Fig. 2 but for quadrupole excitations. 
Fig. 1

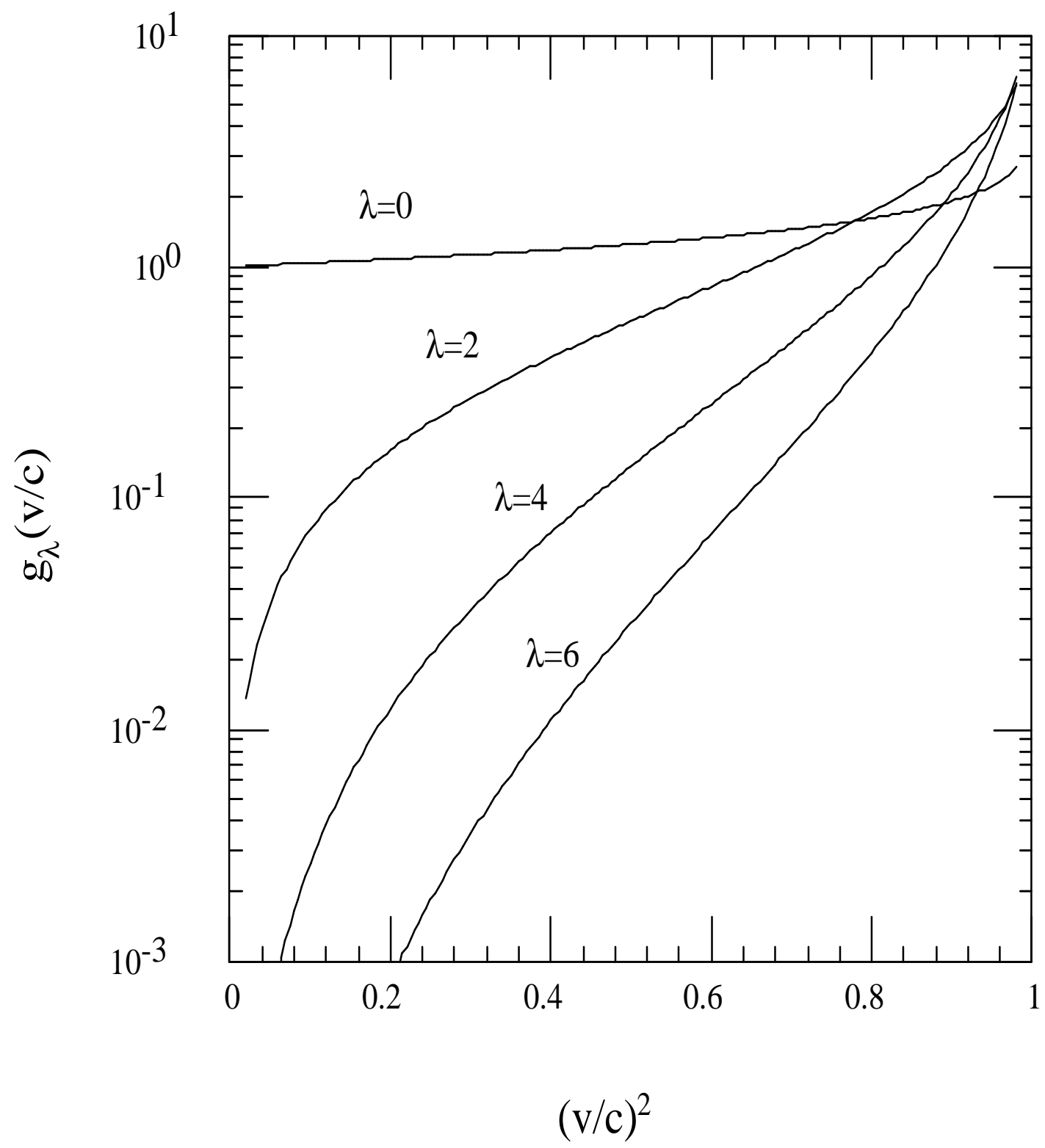


Fig. 2

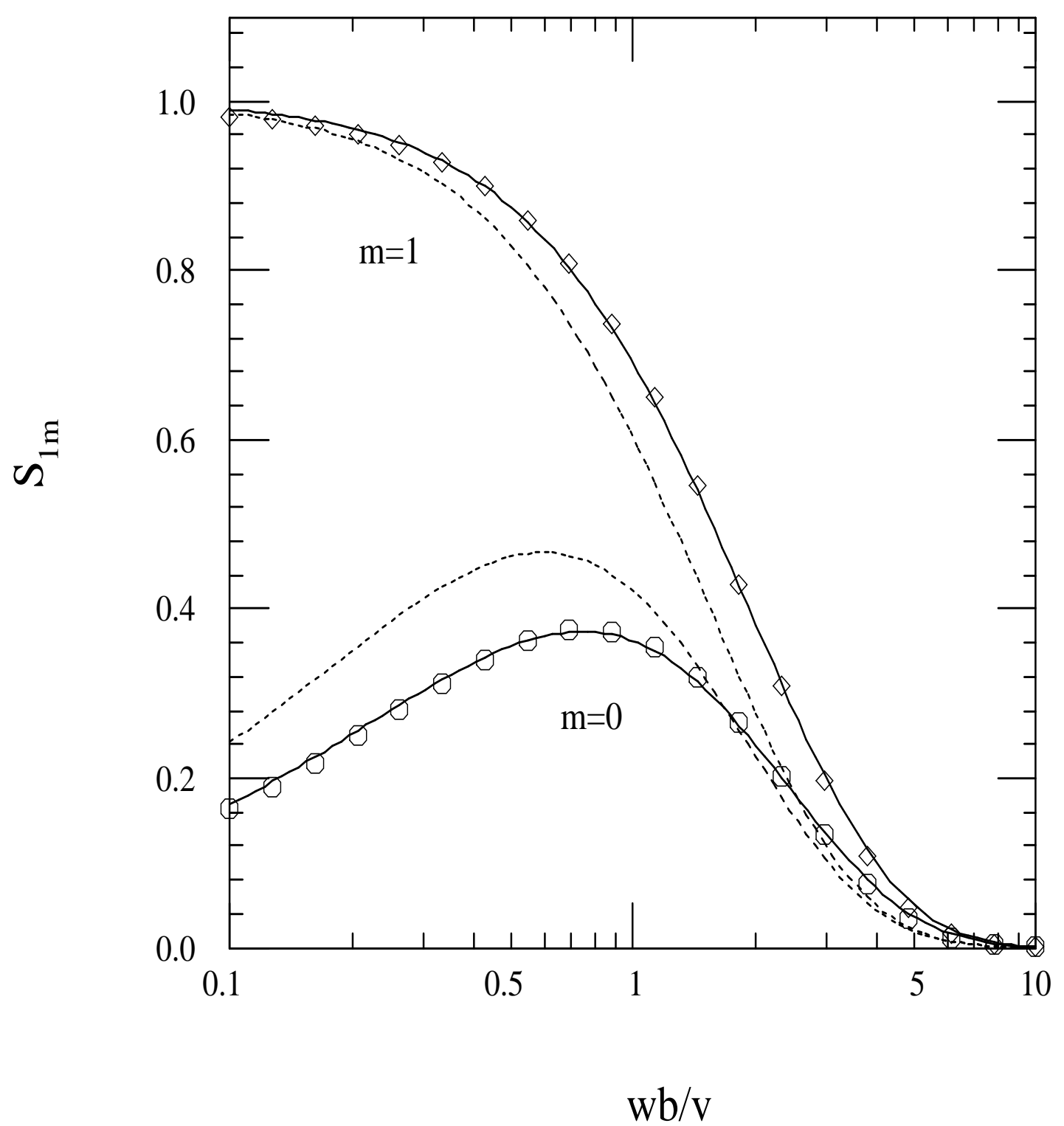


Fig. 3

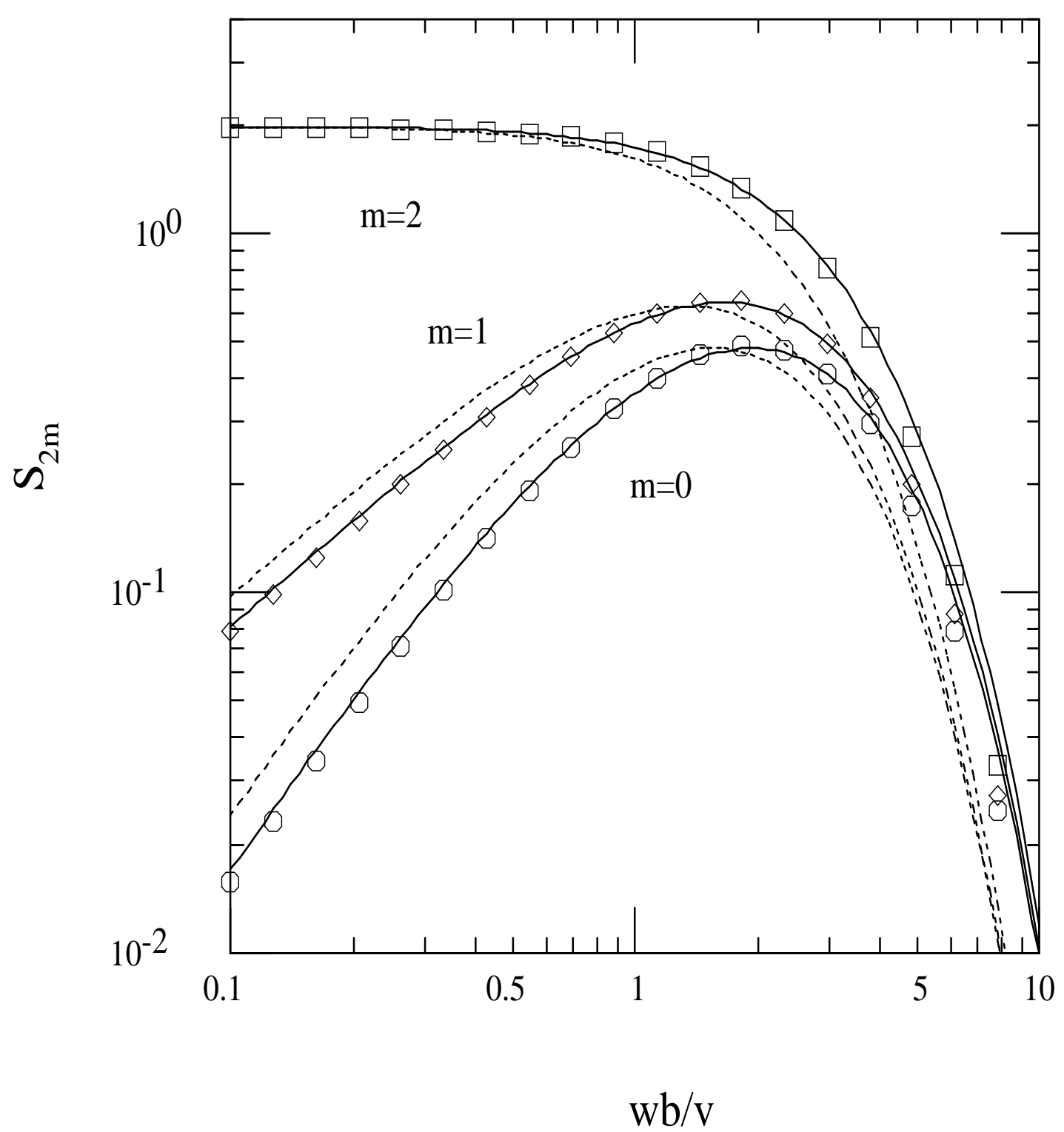

